\title{
Hemolytic Uremic Syndrome
}

National Cancer Institute

\section{Source}

National Cancer Institute. Hemolytic Uremic Syndrome. NCI Thesaurus. Code C75545.

Acute kidney injury associated with microangiopathic hemolytic anemia and

thrombocytopenia. 